\title{
Entreprise moralisatrice et ruralité dans les manuels français de la IIIe république
}

\section{Pierre Guibbert}

\section{(2) OpenEdition \\ 1 Journals}

Édition électronique

URL : http://journals.openedition.org/trema/1881

DOI : $10.4000 /$ trema. 1881

ISSN : 2107-0997

\section{Éditeur}

Faculté d'Éducation de l'université de Montpellier

\section{Édition imprimée}

Date de publication : 1 décembre 1997

Pagination : 125-141

ISSN : 1167-315X

\section{Référence électronique}

Pierre Guibbert, «Entreprise moralisatrice et ruralité dans les manuels français de la Ille république », Tréma [En ligne], 12-13 | 1997, mis en ligne le 01 décembre 1997, consulté le 30 avril 2019. URL: http://journals.openedition.org/trema/1881 ; DOI : 10.4000/trema.1881

Ce document a été généré automatiquement le 30 avril 2019.

Trema 


\title{
Entreprise moralisatrice et ruralité dans les manuels français de la IIIe république
}

\author{
Pierre Guibbert
}

Pour Guy Boisson

1 La silhouette d'un village à l'horizon, un enfant vêtu d'une pèlerine, une paire de galoches qui claquent fièrement sur le chemin empierré, cette scène dynamique illustre le thème de la rentrée des classes sur lequel s'ouvraient la plupart des livres de lectures du Cours élémentaire et du Cours moyen'1.

2 C'est bien connu, l'école primaire de la III république - telle, du moins, qu'on peut l'approcher à travers ses manuels - a valorisé à l'extrême les figures de la ruralité. Or cette surreprésentation est particulièrement sensible lorsqu'on examine les modalités de la moralisation scolaire depuis Jules Ferry jusqu'à la veille de la deuxième guerre mondiale.

3 Il s'agit là d'une énorme question dans la mesure où le discours moralisateur de l'école française a débordé des manuels de morale pour se déployer dans tous les secteurs de l'enseignement. Ainsi les manuels d'arithmétique proposaient aux enfants de clôturer des champs et de planter des vignes à intervalles réguliers, avant de déterminer le bénéfice de la récolte et de calculer combien rapporterait cette somme placée à la Caisse d'épargne $\mathrm{du}$ bourg voisin - et tous ces énoncés, à bien y regarder, ont des connotations morales. Plus directement encore, les manuels de leçons de choses et de sciences appliquées apprenaient aux futurs paysans à fabriquer le compost qui enrichit la terre et posaient des principes d'hygiène et d'économie domestique destinés à améliorer la vie à la ferme. Et, naturellement, les manuels d'histoire participaient à cette entreprise éducative en présentant aux enfants, pour compléter les enseignements délivrés par les grands personnages patentés de l'histoire nationale, des modèles de dévouement civique incarnés par de simples paysans transfigurés par leur foi patriotique. 
Dans ces conditions, j'ai hésité à labourer consciencieusement cet énorme territoire ${ }^{2}$; et j'ai renoncé à définir un corpus scrupuleusement équilibré et à bâtir des hypothèses de travail élaborées... Aussi bien, je vous convie plutôt à une flânerie à travers un domaine qui, avec le temps, m'est devenu assez familier... Mais sachez que j'ai été moi-même assez moralisé - précisément dans une école rurale, et au sortir de la $\mathrm{III}^{\mathrm{e}}$ République - pour ne pas tricher avec les grands principes de l'honnêteté scientifique...

Image $1:$ « La route qui conduit l'enfant à l'école du village est le chemin du Bien. ».

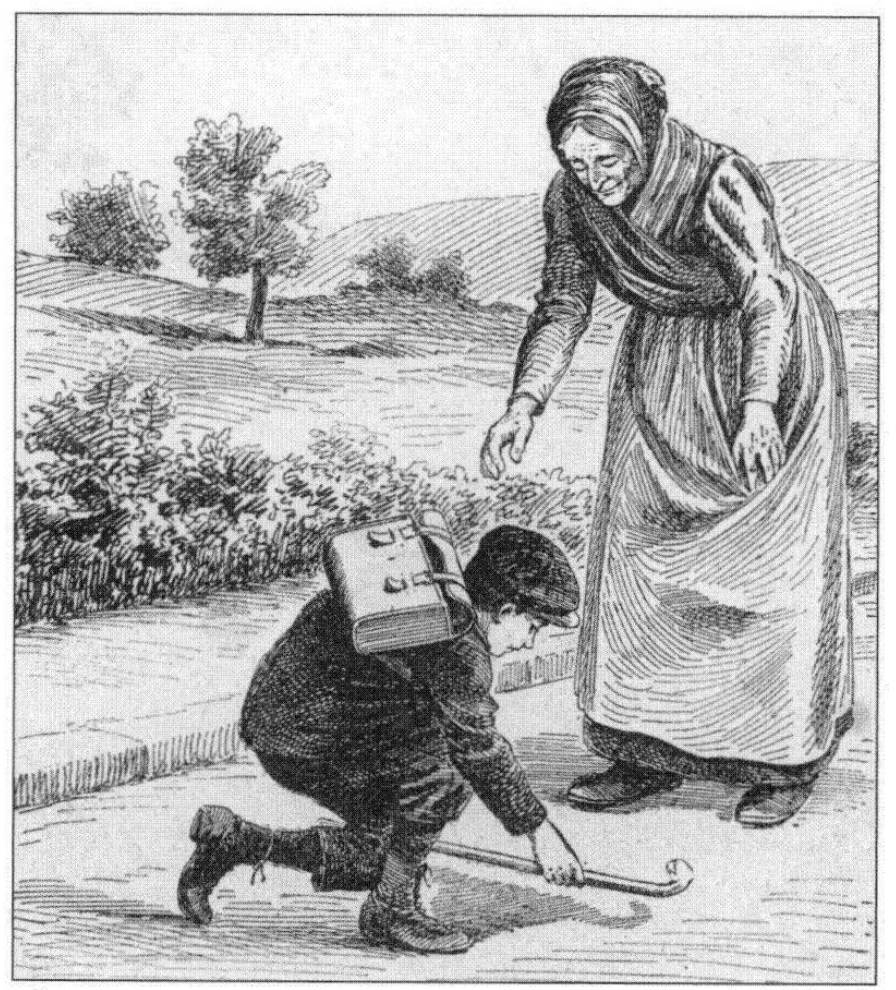

MARTIN J. \& LEMOINE A., Lectures choisies d'auteurs français - Cours moyen et Cours supérieur, Paris, Librairie d'Education Nationale, 1903, 393 p., p. 1.

\section{La bonne campagne}

5 Au premier abord - et ce constat vaut surtout pour les ouvrages les plus anciens et ceux réservés aux enfants les plus jeunes - la campagne morale apparaît comme un lieu édénique.

6 Dans les premiers manuels de la III ${ }^{\mathrm{e}}$ République, la terre est ontologiquement bonne et généreuse, ce qu'expriment de petits poèmes qui se gravent aimablement dans les jeunes mémoires :

"Pour nous que la terre est bonne,

Et que nous la bénissons!

A nos travaux elle donne

Arbres, fleurs, fruits et moissons $"^{3}$

7 Le Trésor moral de l'écolier regorge ainsi de proverbes et de formules imprégnés de ruralité, comme "Bon fermier fait la bonne terre", "manger son blé en herbe " ou "jeter le 
manche après la cognée $\rrbracket^{4}$. Il abonde en maximes du genre "Qui sème bon grain récolte bon pain » ou "Il faut semer pour récolter $»^{5}$.

Il apparait aussi que cette campagne rayonne de beauté. Le mystère pré-romantique de l'harmonie rurale, l'évidence de la splendeur des campagnes françaises en imposent et constituent en eux-mêmes un puissant agent moralisateur : pacifiant, ce spectacle ${ }^{6}$ suffit à communiquer à l'enfant un désir de perfection et l'on retrouve dans cette nature superbe comme des échos laïcisés de la grande tradition augustinienne qui fit de la magnificence de la Création une des preuves fondamentales de l'existence de Dieu?

Cependant, au-delà de ce côté "vicaire savoyard ", la ruralité scolaire se caractérise par une extrême simplicité qui est d'un bon rendement pédagogique auprès de jeunes enfants. Le monde agricole des premiers manuels de la III République - parfois rugueux et austère - est presque toujours pétri de vérité, de réalité, de loyauté. On n'y trouve ni feintes, ni simagrées, ni faux-semblants. Il est, dans tous les cas, parfaitement « lisible». Pour illustrer ce point de vue, les livres de lectures esquissent souvent d'édifiantes confrontations entre la ville artificieuse et la campagne sans apprêts, et ces rencontres pittoresques tournent toujours à l'avantage du représentant du monde rural, souvent un petit paysan, snobé par un enfant de la ville qui se croit à tort supérieur à lui ${ }^{8}$. A ce niveau, la ruralité scolaire répond donc aux vœux de cet illustre Français qui affirmera dans quelques années, après bien d'autres, que « la terre ne ment pas »...

10 Cette bonne et belle campagne est d'essence géographique. Elle a son temple : la ferme idéale, dont les contours vertueux rappellent les utopies élaborées par les physiocrates et les chantres du romantisme social - je pense ici aux «bergeries» de Georges Sand et, surtout, à la ferme normande des Mystères de Paris où se régénère, au sens propre comme au sens figuré, Fleur-de-Marie rongée par les miasmes de la ville ${ }^{9}$. L'exploitation agricole selon les manuels - comme la ferme de M. Morand ${ }^{10}$ donnée en modèle par un instituteur aux élèves de sa classe - est tout ensemble un berceau d'harmonie sociale, puisque les domestiques y font partie de la famille, et un laboratoire des dernières techniques d'élevage et de culture. 


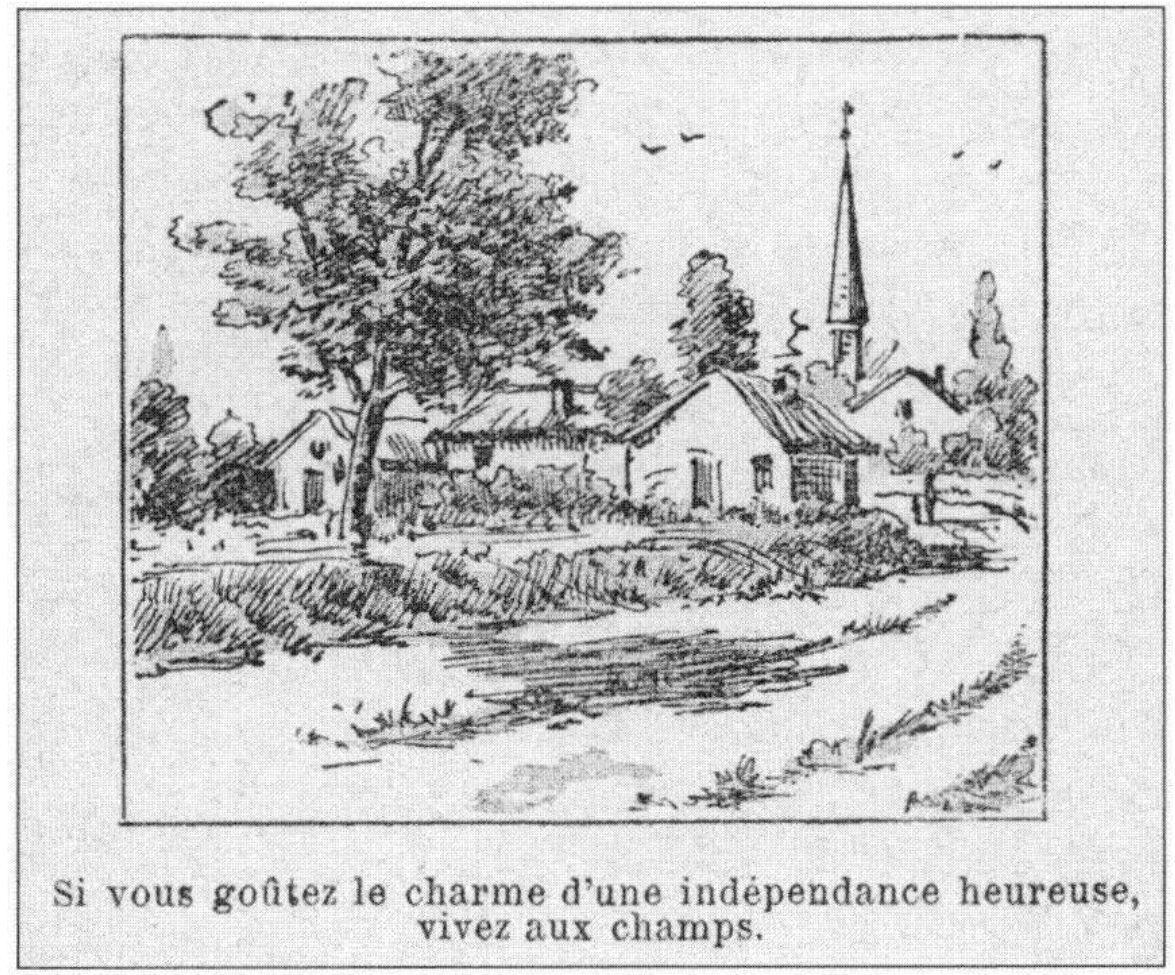

THAUZIÈS [Max ELLYAN], CAZALS, L'écolier ariègeois, récréations morales et littéraires - (Niveau non précisé), Pamiers, Librairie-imprimerie Mme. Delaye, 1900, 344 p., p. 329.

11 Cette modernité ressort - par exemple - de l'extraordinaire correspondance qu'instaure un manuel de lecture entre un parisien condescendant, Paul Rumel, et le bien nommé Jean Terrien, de Saint-Georges-sur-Seugne : les discours de ce solide paysan, qui puise ses arguments dans les ouvrages de vulgarisation de la Bibliothèque des agriculteurs combattent les préjugés du citadin et font valoir que dans la France d'aujourd'hui une ferme bien gérée est une véritable usine, dont les produits assurent l'essentiel de la richesse de la France ${ }^{11}$. Et l'on note, dans le même sens, que les petits orphelins du Tour de la France par deux enfants trouvent la récompense de leur courage et de leur loyauté dans le cadre idyllique de la ferme de la Grand'Lande évoquée à la fois comme une thébaïde et comme une métaphore de la terre patrie ${ }^{12}$.

Dans cette première perspective, la ruralité française abrite quelques vertus spécifiques.

Elle est notamment - par opposition à la ville délétère - le lieu privilégié des familles unies ${ }^{13}$. Elle est aussi le lieu privilégié de la frugalité où se forment des corps sains et vigoureux: selon les manuels de morale, l'alcoolisme est un vice urbain qui frappe "l'ouvrier quand il est fatigué, l'homme de bureau quand il s'ennuie »14. Les bagarres qui dégénèrent en crimes ont lieu sur les trottoirs des grandes villes, aux portes des cabarets. En revanche, les jeunes ruraux, habitués au grand air et aux nourritures saines, illustrent valablement les grands principes hygiénistes en honneur sous la III République : demain, ils feront d'excellents soldats endurants, endurcis par l'habitude du travail manuel parfois dénommé « labeur » ou « besogne $»^{15}$. 
La campagne est en effet par excellence l'école de l'effort saisi dans sa double dimension, physique et morale ${ }^{16}$. Le travail qui ennoblit est le sujet de charmants apologues rimés comme ce dialogue intitulé Les deux charrues :

"Le soc d'une charrue, après un long repos,

S'était couvert de rouille. Il voit passer son frère,

Tout radieux, revenant des travaux.

" Forgés des mêmes bras, de semblable matière,

Lui dit-il, je suis terne, et toi, poli, brillant;

Où as-tu pris cet éclat, mon frère ? - En travaillant $»^{17}$

15 Il fournit aussi le thème de nombreux tableaux aux accents religieux - je pense évidemment aux chromos de Millet - L'homme à la houe et Les glaneuses figurent en bonne place dans les livres de morale enfantine ${ }^{18}$ - et tout particulièrement au tableau de Holbein qui ouvre La Mare au diable de George Sand. Cette scène de labour est souvent reproduite dans les manuels de lecture.

Ici, le travail des champs caractérise le monde rural comme un univers grave, exigeant, sérieux, conséquent, où toute frivolité, toute légèreté se paient comptant et au prix fort ${ }^{19}$. Tel est le sens des mésaventures de Jeannot qui remet à demain le labour de son champ pour assister à un repas de famille. Le lendemain il se ressent d'une indigestion, le surlendemain il pleut à verse, le jour suivant son cheval est indisposé, puis vient un jour de fête qu'il convient de chômer, après quoi surgissent d'autres obstacles comme le décès d'un parent proche... tant et si bien que "lorsqu'il se mit à labourer, la saison de semer était passée : aussi n'eut-il rien à récolter $»^{20}$

\section{La mauvaise campagne}

17 Cependant au fur et à mesure que l'on s'éloigne des origines de la III ${ }^{e}$ République et que l'on s'élève dans le cursus scolaire, ces représentations se heurtent à la grande tradition jacobine qui situe le moteur de l'Histoire et le moteur du Progrès au cœur de la Ville. Apparaît alors une mauvaise campagne qui est d'essence historique : c'est la Vendée des Chouans; ce sont, par extension, tous les territoires arriérés où l'on fait appel aux rebouteux plutôt que d'appeler un médecin ${ }^{21}$, tous les bastions où s'enracinent les résistances têtues aux Progrès de la civilisation, à la République et à la laïcité. Il s'agit d'un héritage ténébreux de l'Ancien Régime ${ }^{22}$, ce qui amène certains pédagogues à faire de cette campagne détestable un phénomène spécifiquement français ${ }^{23}$.

Considérée sous ce nouvel angle, la ruralité nationale est perçue comme un anachronisme et les vertus que lui prêtait la pédagogie traditionnelle semblent inversées. Certes, les paysans courbés sur leur charrue s'échinent au travail, mais ce travail apparaît dégradant: ainsi, le père François, vieilli avant l'âge, se tue au labeur ${ }^{24}$. Surtout, l'obscurité des campagnes entretient d'anciennes superstitions. Les cultivateurs sont alors peints avec des accents naturalistes. Ce sont des primitifs proches de l'animalité, dominés par des instincts sauvages et cruels. Tel villageois brutal roue de coups l'âne chargé de sacs de blé qu'il apporte au moulin ${ }^{25}$. Tel fermier cloue une chouette vivante sur le portail de sa grange. Et, à leur exemple, les petits campagnards multiplient les actes de sadisme envers les animaux.

19 Il serait fastidieux de recenser ici les tristes exploits de ces vauriens qui sillonnent la campagne à la recherche de victimes innocentes : la liste serait trop longue des crapauds 
martyrisés ${ }^{26}$, des chiens auxquels on attache une casserole à la queue, des petits oiseaux dénichés ${ }^{27}$.

Toutes ces turpitudes sont commises par des enfants en rupture de ban. Le mauvais enfant type est "une espèce de vagabond malfaisant [...] Il trompe souvent son père et sa mère en disant qu'il va à l'école. La vérité est qu'il profite de ce que son père, retenu par son travail, ne peut le surveiller, pour aller courir avec d'autres vauriens de son âge. Il déniche les oiseaux, il maraude dans les jardins et les champs; il se rend coupable de toute sorte de méfaits. Le garde champêtre est toujours à ses trousses. ». Bref, ce mauvais sujet est un mauvais élève, un adepte de l'école buissonnière ${ }^{28}$ qui cède aux attraits d'une liberté fallacieuse comme ce berger insouciant une sorte de Gaspard Hauser - rencontré par Ernest Lavisse au cours d'une de ses promenades dans la forêt de Thiérache ${ }^{29}$.Car le défaut de connaissances, davantage encore que la paresse, est mère de tous les vices.

Dans ce contexte de brutalité, on peut en effet mourir, ou tuer par mégarde son père et sa mère. Ainsi le petit Thomas, qui n'a pas appris à lire, verse à son père alité une potion contenue dans un flacon dont l'étiquette rouge mentionne " poison, pour usage externe » et il manque le tuer ${ }^{30}$. Ainsi un instituteur, prenant sans doute appui sur un fait réel, lance à ses élèves cette terrible mise en garde : «Et ces pierres que vous aimez tant lancer, que de maux elles ont causé! Il est arrivé à un malheureux enfant d'atteindre ainsi sa mère qu'il ne voyait pas et de la tuer. $»^{31}$

Tel est le cadre, tels sont les enjeux du combat titanesque que les instituteurs ruraux livrent passionnément, déployant des trésors de pédagogie pour que, à terme, les rayons du Progrès dissipent peu à peu les ténèbres.

Image 3 : « Victor est un vaurien. »

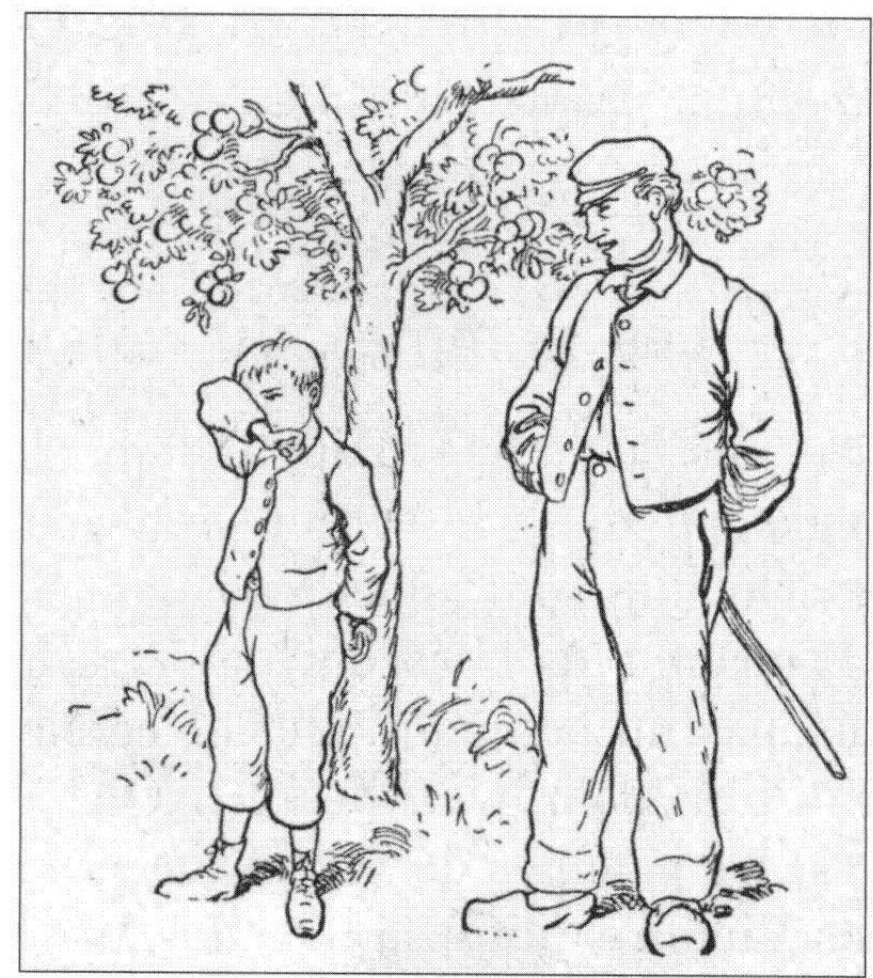

PIERRE A., LETRAIT L. \& Mme BODIN Z., Lectures et récitations morales - Cours élémentaire, Paris, $\mathrm{F}$. Nathan, $8^{e}$ éd., 1909, 160 p., p. 114. 


\section{Le recours urbain} Heureusement, les maîtres de la $\mathrm{III}^{\mathrm{e}}$ République sont aidés dans cette tâche par des hommes de bonne volonté : authentiques savants, éclairés par une instruction supérieure, ou, plus simplement, citoyens qu'habite une solide conscience républicaine. Ainsi, le père Thomas, qui « s'était fait une spécialité de prêcher aux familles et aux enfants", fait la leçon à un fermier voisin qui avait dispensé son garçon d'école sous prétexte qu'il n'est pas besoin d'être instruit pour être un bon laboureur ${ }^{32}$. Or le père Thomas est un ancien ouvrier, et l'on remarque ici que le discours du Progrès vient essentiellement de la ville où réside une bourgeoisie instruite, dotée d'une conscience morale et historique supérieure. C'est la ville qui délivre au cours de ses visites - j'allais dire de ses inspections - des certificats de bonne ou de mauvaise conduite. C'est elle qui surveille, récompense et punit. Le mauvais paysan de la Ferme des deux épis, par cupidité, met-il de la craie dans son beurre? Il est bientôt confondu par un chimiste en vacances qui, saisi de soupçons, mêle du vinaigre au beurre frelaté et constate que les particules de craie entrent en effervescence ${ }^{33}$. En revanche, l'excellent fermier Bernard - un brave paysan honnête, laborieux et ouvert aux idées nouvelles - reçoit un témoignage de satisfaction mérité de la part de son cousin André Lebon, un professeur de Lyon qu'il a invité à passer l'été à Egriselles ${ }^{34}$.

24 En ce sens, le dialogue qui s'instaure entre la ville et la campagne reproduit le dialogue ordinaire entre le maitre et ses élèves. C'est une communication de haut en bas, procédant d'un paternalisme vigilant, comme il apparaît dans la préface du manuel de lecture de Forsant et Dudouit : "Les textes et les gravures que nous avons rassemblés ici sont l'œuvre d'écrivains et d'artistes remarquables...; Ils ont longuement observé et profondément aimé la nature et les campagnards. Ils seront vos guides [.. . ] $\|^{35} \mathrm{~A}$ ce niveau, la parole moralisatrice de l'école républicaine inverse donc la rhétorique traditionnelle de l'Eglise catholique qui, dans la lignée de Vélez de Guevara, ne prononce l'éloge de la campagne que pour flétrir la ville. Elle inverse ce discours ou plutôt le dépasse puisqu'elle met ces deux espaces en communication afin de ménager les conditions d'une fructueuse évolution. Cet échange ville-campagne est d'ordre matériel, géographique: de ce point de vue, le fait que certains livres de morale fassent l'éloge du chemin de fer et du télégraphe n'est pas une incongruité mais une commodité pédagogique qui facilite les confrontations édifiantes. Mais cette communication est aussi d'ordre historique et spirituel : la ville est en effet le destin des meilleurs enfants des campagnes. Ainsi, les excellents élèves des écoles rurales, sur proposition de l'inspecteur primaire, qui les signale à l'Inspecteur d'Académie, reçoivent une bourse pour aller étudier à la ville et devenir des bourgeois à force d'instruction.

Toutes ces tendances sont cristallisées dans une scène clé, qui porte tant de sens qu'elle est décrite ou représentée dans les manuels de toutes les disciplines - morale, sciences naturelles, leçons de choses, lecture et histoire : il s'agit de la rencontre de Jupille, le petit berger héroïque venu de la montagne, et du savant Pasteur, qui réside à Strasbourg.

Au fond, la ville apparaît comme le territoire des adultes alors que la campagne est un lieu éminemment enfantin. Il existe en effet une correspondance assez exacte entre l'irresponsabilité originelle des enfants et la sauvagerie ancestrale du paysan dont les comportements sont souvent irrationnels, inconséquents. Il y a en effet quelque chose de puéril dans le comportement de ces fermiers qui chassent les moineaux de leurs champs 
ensemencés de peur qu'ils ne dévorent les graines avant de découvrir, un peu tard, que les insectes ont dévoré les grains et rongé les fruits de leurs pommiers ${ }^{36}$.

C'est profondément ce pourquoi l'école privilégie la campagne par rapport à la ville : la campagne est pour l'instituteur une véritable terre de mission.

\section{Un théâtre pédagogique}

Cette sollicitude pédagogique a conduit ${ }^{37}$ les auteurs des manuels à aménager la campagne française pour en faire le théâtre idéal de la moralisation des écoliers.

L'école de la III république a dramatisé, non sans roublardise, l'autonomie du petit rural. D'une part, toutes les possibilités d'action octroyées aux enfants des campagnes font de l'espace rural un terrain de jeux particulièrement séduisant, voire un terrain d'aventures. Mais, en contrepartie, cette liberté romanesque a pour corollaire un libre-arbitre qui fonde la responsabilité morale des écoliers.

Ainsi, tout au long du chemin qui mène de la ferme à l'école, les enfants des campagnes sont soumis à des tentations d'autant plus fortes qu'ils sont livrés à eux-mêmes. Ils sont donc nombreux à céder à leurs pulsions, comme le gros Victor qui chaparde des pommes dans un verger ${ }^{38}$ ou comme le petit Pierre, âgé de huit ans, qui accumule les bêtises : tantôt ce garnement met le feu pour jouer à des herbes sèches; d'autres fois, grimpant aux arbres pour détruire des nids de bouvreuil, il fait des accrocs à sa veste ; ou bien il mutile un jeune cerisier pour manger ses fruits jusqu'au jour où, blessé par un caillou lancé par un de ses camarades, il manque mourir ${ }^{39}$.

31 Naturellement, cette liberté qui vaut pour le pire vaut aussi pour le meilleur, comme en attestent les rapports qui motivent l'attribution des prix de vertus. Les pédagogues raffolent de ces historiettes dénuées d'artifices littéraires, circonstanciées comme des rapports de gendarmerie, convainquantes comme la réalité.

Certaines lectures morales, formées en recueils, disent simplement les petites bonnes actions quotidiennes qui, répétées, fondent les grands mérites. C'est le cas de ces braves garçons qui saisissent toutes les occasions de soulager leurs parents dans les travaux de la ferme ${ }^{40}$. Cependant d'autres récits, plus mouvementés, saluent le sang-froid de Roger et de sa petite sœur Marie qui échappent à la charge d'une vache furieuse poursuivie par des paysans armés de bâtons ${ }^{41}$; ou bien, sortant du registre de la fantaisie, ils célèbrent le courage de précoces héros comme le petit Joseph Serre de Gimons, dans le Gers, qui sauve deux enfants tombés en jouant dans un puits; ou le berger Jupille qui soustrait des écoliers aux morsures d'un chien enragé non loin de Villers-Farlay, dans le Jura ${ }^{42}$. 
Image 4 : « Les enfants des campagnes apprennent le Bien dans le grand livre de la Nature. »

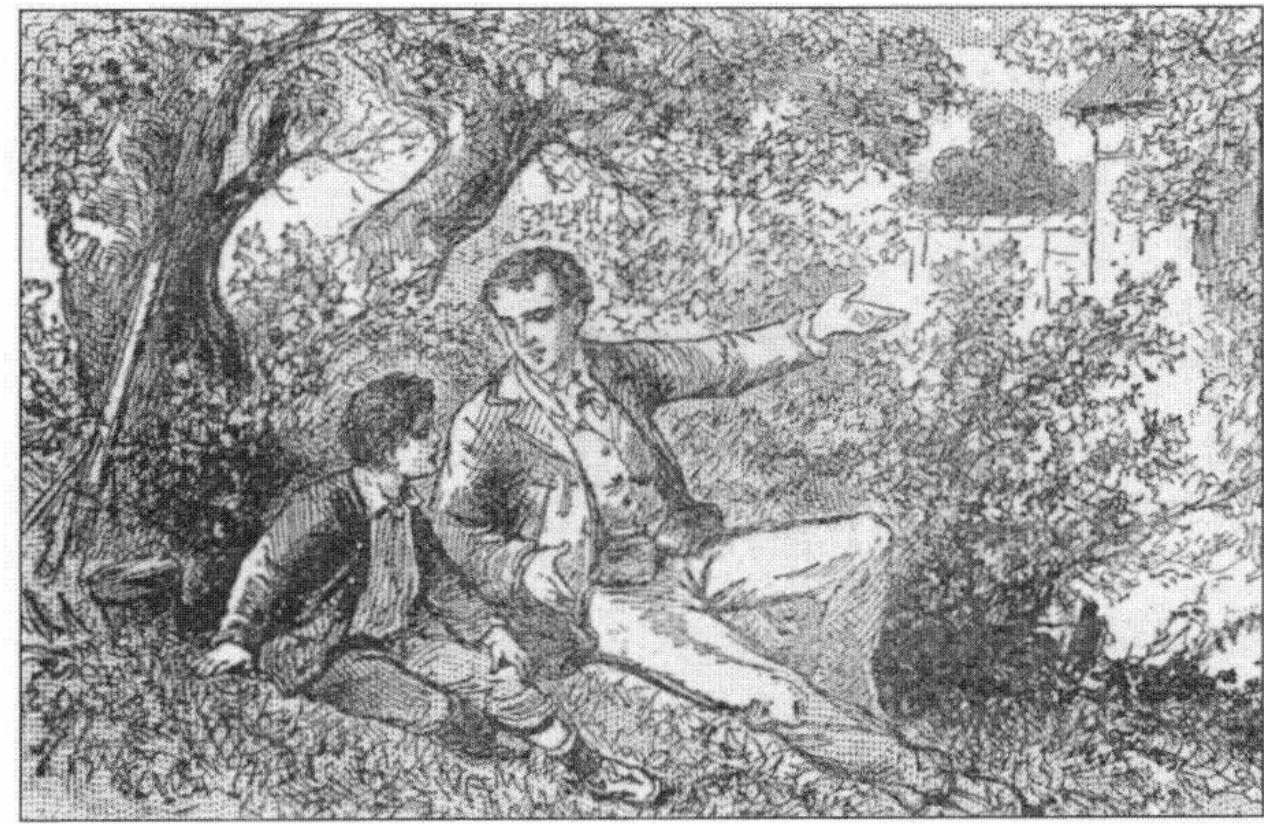

DEVINAT E., Livre de lecture et de morale - Cours élémentaire, Paris, Larousse, 15e éd., 1917, 167 p., p. 44

33 Cette mise en scène du bien et du mal est mise en valeur par les dimensions réduites de la scène rurale. Cet espace est à la mesure des enfants, ce qui le rend particulièrement propre à l'illustration de notions vastes et abstraites comme la patrie ou la solidarité sociale qui, selon les instructions officielles, doivent être abordées dès le cours élémentaire.

A ce niveau l'idée de patrie est abordée par le biais d'une pédagogie sensible et graduelle où l'on va, par paliers, du proche à l'éloigné, du familier à l'inconnu. Dans ces progressions, le village - qui est une entité de taille raisonnable, qui peut être saisi dans son ensemble par le regard de l'enfant, qui est aisément symbolisé par la chère silhouette d'un clocher - tient lieu de moyen terme entre le toit de la maison familiale et le territoire national :

Dis quelle est ta patrie?
C'est la maison de ma naissance
Qui vit mes premiers pas tremblants.
Elle a protégé mon enfance,
La maison de mes chers parents.
Dis quelle est ta patrie?
C'est le joli petit village,
L'école blanche au rouge toit,
Où les maîtres de mon jeune âge
Me disent : "Sois sage, instruis-toi "
Dis, quelle est ta patrie?
Oui, c'est surtout ma chère France
La terre belle et douce aux yeux
Qui met en moi son espérance,
C'est la terre de mes aïeux ${ }^{43}$


lémentairement, mais dans un registre plus terre-à-terre, les devoirs civiques qui se rattachent à la notion de patrie sont heureusement rapprochés du droit de propriété ${ }^{44}$ auquel on dit que les paysans sont particulièrement sensibles. Ainsi, dans une leçon engagée intitulée "Réfutation du cosmopolitisme", deux moralistes insistent sur le caractère concret que doit revêtir l'enseignement du patriotisme : le citoyen ne se bat pas pour une chimère, observent-ils, il protège sa propriété personnelle ${ }^{45}$. Dans cette perspective, les figures familières de la petite patrie - quelques arpents de terre cultivés, un potager, la maison familiale - donnent une apparence convaincante au «beau visage de la patrie $»^{46}$ et préparent mieux que de pompeux discours les enfants des écoles à devenir un jour les acteurs de la Revanche. individualisme et son égoïsme originels pour concevoir la solidarité communautaire et la complémentarité économique qui doivent unir les citoyens de la France moderne. Pour illustrer cette interdépendance les manuels s'appuient généralement sur une symbolique éprouvée en évoquant la production du pain qui met en jeu - dans le cadre familier du petit village - des personnages proches : le paysan, le meunier et le boulanger ${ }^{47}$.

\section{Un agent moralisateur}

37 Mais la campagne pédagogique est bien plus qu'un décor aimable ou dramatique, c'est aussi un puissant agent moralisateur. Dans la lignée du rousseauisme, la campagne est en effet, par elle-même, une grande pourvoyeuse de leçons.

Pour les tout petits elle ouvre le grand livre de la nature, poétiquement illustré, qui offre toute une gamme de situation suggestives capables d'initier ce qu'on appellerait aujourd'hui un « auto-aprentissage $»^{48}$.

La faune est le principal acteur de cette étourdissante animation. Fanor, l'infatigable chien de berger, si dévoué à son maître, fournit au petit écolier un modèle de conscience professionnelle. L'abeille industrieuse lui rappelle les règles de la bonne conduite et le secret de la réussite scolaire qui se fonde sur la diligence et la constance dans l'effort ${ }^{49}$ :

"Jamais d'école buissonnière

Dit cette bonne conseillère

Qui voltige entre terre et ciel " $^{50}$.

40 Le petit oiseau, ${ }^{51}$ qui préfère les bocages àla cage dorée, lui enseigne que la liberté est le bien le plus précieux ${ }^{52}$. La poule qui protège sa progéniture menacée par un chat agressif ${ }^{53}$ , la perdrix traquée par le chasseur, qui se sacrifie pour sauver ses petits, illustre la force de l'amour maternel ${ }^{54}$. Et la tragique mésaventure du petit lapin désobéissant apprend qu'il faut écouter les conseils des parents ${ }^{55}$.

41 La flore aussi, se met de la partie. Ainsi la structure de l'églantine, qui protège ses jolies fleurs par de aides épines démontre qu'il faut savoir dépasser les apparences ${ }^{56}$, Le robuste tuteur soutenant le frêle arbrisseau fait valoir la nécessité de la discipline ${ }^{57}$. Quant au modeste sorbier, il dit les bienfaits de l'instruction à travers un apologue bien venu: cheminant à travers champs, un conscrit fait halte sous un sorbier. Il goûte un fruit vert qu'il trouve détestable. A quelque temps de là, rentrant chez lui en permission, il s'arrête au même endroit et trouve les sorbes mûres et tendres, ce qui fit naître le dicton : « Tu feras peut-être comme la sorbe: de vaurien tu deviendras bon $\|^{58}$. 

l'instituteur sont irremplaçables, surtout lorsqu'on s'élève dans l'échelle de la scolarité. Malheur à l'enfant qui n'en profite pas, sa négligence peut le conduire au bord de la mort à l'exemple de Jacques le paresseux qui, oubliant les leçons de son maître, s'est abrité sous un grand chêne, un jour d'orage. "Le tonnerre gronde, les éclairs brillent, la pluie tombe par torrents » aussi Jules, le bon élève, lui dit : « Ne reste pas là [...] Notre instituteur, dans une leçon de physique, nous a appris qu'il est dangereux, quand il tonne, de se mettre à couvert sous les arbres, car les pointes des arbres ou des édifices attirent la foudre». Et il finit non sans mal à convaincre le malheureux de fuir avant que le grand arbre soit anéanti ${ }^{59}$.

\section{Les méchants doivent être punis}

Lorsqu'elle met en jeu sa légitimité, l'école primaire de la $\mathrm{III}^{\mathrm{e}}$ république peut donc développer une stratégie de la terreur qu'elle " emprunte » à la tradition pédagogique de l'Eglise catholique.

Dans cette veine, les transgressions aux règles de la morale élémentaire débouchent souvent sur une sanction immanente. Ainsi, Marc est cruellement mordu par une oie à laquelle, par désœuvrement, il avait donné des coups de bâton ${ }^{60}$. Ainsi l'âne Mirolu, caressé par la pointe d'un couteau, se venge en désarçonnant le méchant De Cherville ${ }^{61}$. Ainsi le petit Jeantou, qui prétendait dénicher un pauvre oiseau pour éblouir son amie Linou se blesse légèrement en tombant de l'arbre et, de plus, il est sévèrement rabroué par la petite fille compatissante ${ }^{62}$. Encore, ceux-là s'en tirent à bon compte, mais d'autres, qui ont enfreint les interdictions parentales, sont plus durement touchés. Louis, qui cache sa main entortillée dans un linge, a voulu scier un bout de bois ${ }^{63}$; Charles, huit ans, qui jouait sans autorisation dans une vieille carrière, fait une chute grave qui le laissera estropié ; et Jules, pour avoir bu de l'eau trop fraîche en période estivale, est frappé par une fluxion de poitrine dont il mourra. Lieu naturel et archaïque, la campagne convient particulièrement aux manifestations d'une justice à l'ancienne, qui apparait sans doute d'autant plus redoutable à l'enfant qu'il n'a aucune possibilité de s'y soustraire - alors qu'il peut espérer, en se dissimulant, déjouer la vigilance du garde-champêtre. Elle est d'autant plus à craindre qu'elle est souvent terrible dans son immédiate simplicité - on est puni par où l'on a péché, ce qui est apprécié par des enfants qui appliquent entre eux, quand ils le peuvent, l'antique loi du Talion. ${ }^{64}$

Grosso modo, cette pédagogie rudimentaire est mise en œuvre dans les petites classes. Audelà, tenant compte des progrès des enfants, l'école joue de préférence sur d'autres ressorts en s'efforçant de motiver ses commandements. Dans ce sens, les instituteurs multiplient les appels qui touchent à la fois le cœur et la raison : "Quand vous aurez envie de tourmenter des insectes, d'arracher les ailes ou les pattes à une mouche, de lancer des pierres aux oiseaux, représentez-vous les souffrances horribles que vous leur infligez " ${ }^{65}$. Dans le même sens, ils encouragent les réflexions anthropomorphiques : «Si on détruit les nids, on rend les oiseaux bien malheureux. Que dirait le petit dénicheur si des méchants détruisaient, démolissaient la maison de son père, et si, lui, on l'enfermait pour le mettre en prison? Et bien, quand le petit dénicheur prend les nids des oiseaux, ou qu'il en enlève les petits pour les enfermer dans une cage, il commet une mauvaise action. ${ }^{66}$. Cependant d'autres, plus pragmatiques, spéculent sur l'intérêt bien compris. On a établi qu'une nichée de mésanges, nourrissant jusqu'à vingt petits, détruit en une vingtaine de jours, quinze mille chenilles et, en un an, plus d'un demi-million d'insectes. Or le même calcul vaut pour le hibou et le chat-huant. Certes 
"[...] ils sont franchement laids, et leur cri est bien désagréable, quand il retentit dans les ténèbres. Mais ils font une guerre terrible aux rats, aux souris et aux mulots qui ravagent nos champs et nos greniers. $1^{67}$

Image 5 : «Sanction immanente : Marc mordu par l'oie qu'il martyrisait. »



JOST C. \& HUMBERT V., Lectures pratiques, Education et instruction, Leçon sur les choses usuelles Cours élémentaire et cours moyen, Paris, Hachette et Cie, 22e éd., 1908,182 p., p. 112.

Enfin, au cas où ce travail de prévention s'avérerait insuffisant, la mauvaise action est sanctionnée par une punition infligée par le père de famille ou un représentant de l'autorité de l'état.

Lorsqu'il bat la campagne, l'enfant n'est jamais seul avec sa conscience. Un réseau serré de sociabilité le protège, notamment de lui-même. Dans son paradis rural, il est en permanence sous le regard de l'adulte à qui rien ou presque n'échappe, comme Adam et Eve étaient sous le regard de $D_{i e u}{ }^{68}$, et sa merveilleuse liberté n'est qu'une mise à l'épreuve. Aussi bien l'écolier qui s'apprête à chaparder des pommes doit toujours imaginer, en embuscade derrière chaque haie, le propriétaire du verger, un voisin, ou le garde-champêtre qui surgira lorsqu'il s'y attend le moins, avec ses grosses moustaches et sa plaque bien astiquée ${ }^{69}$. C'est lui qui interpelle le chenapan d'une voix de stentor avant de le traîner par l'oreille jusqu'à ses parents, jusqu'au propriétaire spolié, voire, dans les cas graves, jusqu'au maire du village qui prononcera la sentence... ${ }^{70}$. En dernière extrémité, les gendarmes - qui sont des figures rurales de l'autorité - dresseront procèsverbal au nom de la loi ${ }^{71}$. Certains manuels citent in extenso les articles de la loi Grammont qui stipule que "seront punis d'une amende de 5 à $15 \mathrm{~F}$. et peuvent l'être de un à deux jours de prison ceux qui auront exercé publiquement et abusivement de mauvais traitements aux animaux domestiques $»^{72}$ et un ouvrage va jusqu'à rappeler que "Jusqu'à l'âge de seize ans, un ordre du père de famille suffit pour faire mettre un enfant en prison pendant un mois ». 


\section{Une morale de la virilité} substitue à l'enseignement religieux qui ouvrait traditionnellement la journée de l'écolier. Or dans les catéchismes diocésains, l'exposé de la doctrine - le corps intemporel des vérités à croire - était souvent suivi d'une partie morale conséquente, plus «mondaine » et plus réaliste ${ }^{73}$, qui inscrivait les valeurs chrétiennes dans un contexte contemporain. La morale laïque des débuts de la III République doit beaucoup à cet héritage reconnu et même - n'en déplaise à ceux qui campent sur des stéréotypes idéologiques - revendiqué par les grands fondateurs de l'école publique, les Félix Pécaut, les Jules Payot, les Ferdinand Buisson qui avaient trop de culture historique et trop de conscience historienne pour se laisser aveugler par les impératifs immédiats de la lutte anti-cléricale.

49 A l'enseignement de la morale religieuse elle emprunte donc son rituel et ses formes pédagogiques: la lecture magistrale d'un récit édifiant suivie d'un bref commentaire destiné à établir le sens et de la formulation du précepte du jour, que les élèves calligraphient sur leur cahier de classe. Et, bien sûr, elle partage avec lui nombre de valeurs.

Cependant, à y regarder de plus près, la morale enseignée par les manuels de la III $^{\mathrm{e}}$ République apparaitt nettement plus abrupte et plus virile que sa concurrente directe, la morale catholique qui continue à être enseignée par les prêtres, le jeudi matin. garçons parce qu'elle est fondamentalement une morale sociale. Les filles, qui ne font pas l'école buissonnière, les filles qui - à la ville comme à la campagne - ne s'éloignent guère des jupes de leurs mères, ne présentent que des défauts individuels tels que nonchalance, gourmandise, étourderie, mensonge, négligence, égoïsme, coquetterie ou médisance : ceux-là même qui, précisément, focalisent l'attention de la pédagogie religieuse. Quant à leurs vertus, elles ne se haussent pas au-dessus du quotidien. La compassion qui pousse la petite Fanchon à nourrir les petits oiseaux ${ }^{74}$ ne pèse pas d'un grand poids au regard de l'héroïsme d'Henri, qui sauve sa sœur de la noyade ${ }^{75}$ !

les grandes vertus que s'attache à promouvoir l'école de la III ${ }^{e}$ République ne sont pas d'ordre domestique mais social : il s'agira, demain, d'être un travailleur consciencieux, un soldat courageux et un citoyen instruit, attaché à la démocratie.

53 Dans ce cadre exigeant, la ruralité chimérique façonnée par les manuels de la $\mathrm{III}^{\mathrm{e}}$ république aura fourni aux instituteurs tout ce dont rêvent un enseignant habité par la vocation pédagogique : un public idéalement ignorant sur une terre vierge. 


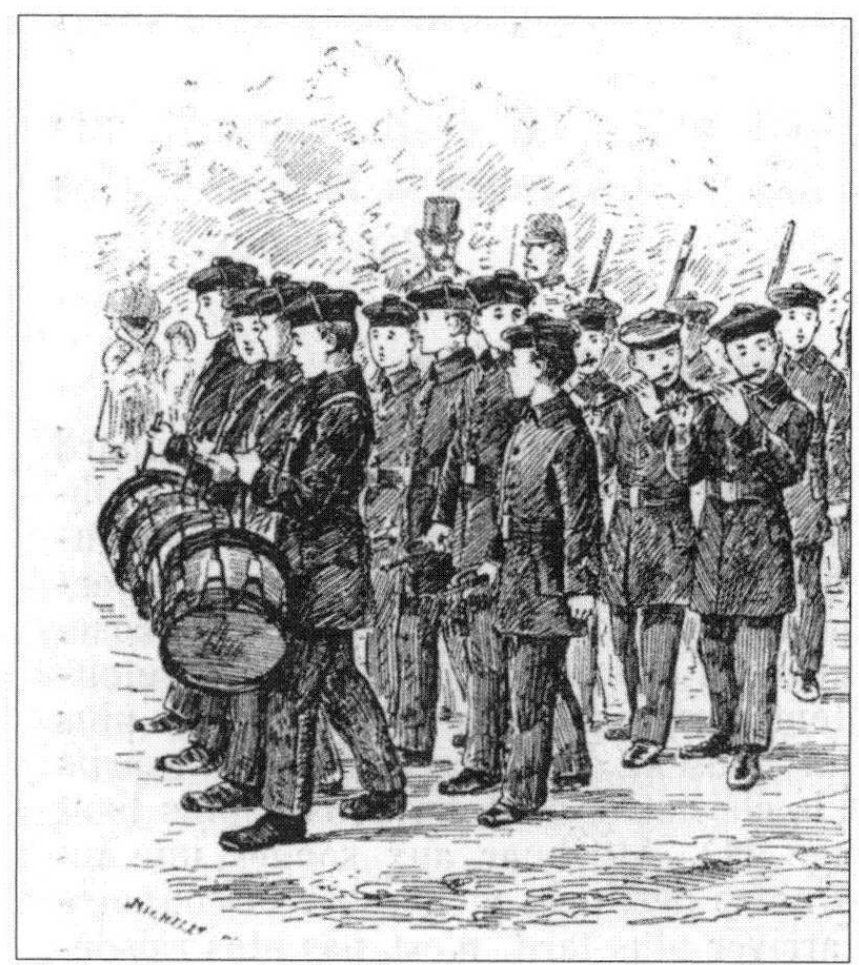

LEBAIGUE C., Le livre de l'école - Cours préparatoire, Paris, Éditions Eugène Belin, 15e éd., 1885, 114 p., p. 70.

\section{NOTES}

1. Cf., par exemple, PIERRE A., LETRAIT L. \& $\mathrm{M}^{\mathrm{me}} \mathrm{BODIN}$ Z., Lectures et récitations morales - Cours élémentaire,Paris, F. Nathan, 8e éd., 1909,160 p., p. 38. MARTIN J., LEMOINE A., Lectures choisies d'auteurs français - Cours moyen et Cours supérieur.Paris, Librairie d'Education Nationale, 1903, 393 p., p. 187. DEVINAT E., Livre de lecture et de morale - Cours élémentaire,Paris, Larousse. $15^{\mathrm{e}}$ éd., 1917, 167 p., p. 54 etc.

2. Le Centre de Documentation en Histoire de l'Education de l'I.U.F.M. de Montpellier conserve quelques 3870 manuels de la III ${ }^{\mathrm{e}}$ république.

3. JOST C. \& HUMBERT V., Lectures pratiques, Éducation et instruction, Leçons sur les choses usuelles Cours élémentaire et Cours moyen,Paris, Hachette et C $C^{\mathrm{ie}}, 22^{\mathrm{e}}$ éd., 1908, 182 p., p. 48.

4. Ouvrage Coll. (Plusieurs instituteurs), Trésor moral de l'écolier - Cours élémentaire et Cours moyen.Paris, Librairie Larousse, vers 1890, 117 p., p. 28.

5. JOST C. \& HUMBERT V., op. cit.note 3. p. 55 et p. 60.

6. $\mathrm{M}^{\text {me }}$ DEPUICHAULT A., Les récits de mon oncle, histoires récréatives et morales - Niveau non précisé. Limoges, Paris, F. F. Ardant frères, vers 1880, 205 p., p. 107.

7. GUIBBERT P., «Le petit monde des manuels d'apprentissage de la lecture », in Internationale Schulhuchforschung. Frankfurt, Diesterweg, octobre 1997, 288 p., pp. 14-23. 
8. GÉRARD J., Maximes morales du petit écolier français - Cours élémentaire et Cours moyen.Paris, Gedalge jeune, $2^{e}$ éd., 1892, 160 p. Programme 1882, p. 70.

9. GUIBBERT P., "Quand le cinéma fait l'éloge de la campagne", in Champ contre-champs, le cinéma rural en Europe,Paris, Centre Georges Pompidou, collection Cinéma singulier, 1990, pp. 72-76.

10. GÉRARD J., Maximes morales de l'écolier français - Cours moyen et Cours supérieur, Paris, Gedalge jeune. $8^{\mathrm{e}}$ éd., 1883, 308 p. Programme 1882, p. 66.

11. FORSANT O. \& DUDOUIT P., La vie aux champs, choix de lectures pour les écoles rurales - Classe de fin d'études, Paris, Larousse, 1930, 272 p.

12. Cf.GUIBBERT P., " Discours didactique et prétexte romanesque dans Le Tour de la France par deux enfants ", in Manuels et apprentissages au fil des siècles,Montpellier, Université Paul Valéry, I.U.F.M. de l'Académie de Montpellier. Collection Discours Didactiques, 1996, 110 p., pp. 63-84.

13. ESTIENNE P. \& DANIEL H., Deuxième livre de récitation et de morale-Cours moyen, Paris,Larousse, $9^{e}$ éd., 168 p., p. 19. BRÉMOND E. \& MOUSTIER D., L'Education morale et civique à l'école-Cours moyen et Cours supérieur,Paris, Librairie Delalain, $10^{\mathrm{e}}$ éd., 1920, 320 p., pp. 27 et 28 etc.

14. BRÉMOND E. \& MOUSTIER D., op. cit.note 13, p. 119 ; PAYOT J., La morale à l'école - Cours moyen



15. Pour un développement de cette perspective, voir. GUIBBERT P. \& RIEMENSCHNEIDER R., chapitre "Former les corps" in La guerre des écoles.Braunschweik. Montpellier, Georg-Eckert Institut. I.U.F.M. de Montpellier, (àparaître).

16. BAUDRILLARD J. \& KUHN M., op. cit.note 3, pp. 164-165.

17. JOST C. \& HUMBERT V., op. cit.note 3. p. 47.

18. Par exemple dans FORSANT O. \& DUDOUIT P., op. cit.note 12, pp. 209 et 257.

19. Cf.PAYOT J., op. cit.note 14 ; BAUDRILLARD J. \& KUHN M., op. cit.note 16 : MARTIN J. \& LEMOINE A., op. cit.note 1, p. 187 ; etc.

20. VESSIOT M., Cours et méthode d'enseignement moral - Niveau non précisé,Paris, E. Belin, $2^{\mathrm{e}}$ éd.. 1897, 271 p., pp. 160-161.

21. PAYOT J., op. cit. note 14. p. 49.

22. Cf.BRÉMOND E. \& MOUSTIER D., op. cit.note 13, p. 2 ; PAYOT J., op. cit.note 14, p. 199 ; etc.

23. Ils le font par exemple en opposant - comme PAYOT J., op. cit.note 14. p. 51 - l'état d'arriération de la paysannerie française à l'intelligence de la paysannerie danoise.

24. DEVINAT E., op. cit. note 1, p. 16.

25. GÉRARD J., op. cit. note 8, p. 139.

26. BAUDRILLARD J. \& KUHN M., op. cit. note 16, p. 203.

27. Cf.ESTIENNE P. \& DANIEL H., op. cit.note 13, p. 83 ; BRÉMOND E. \& MOUSTIER D., op. cit.note 13 , p. 15 ; BAUDRILLARD J. \& KUHN M., op. cit.note 16, p. 61 et 260 etc.

28. CAZES E., Instruction morale et civique - Cours moyen,Paris, Ch. Gedalge jeune, $7^{\mathrm{e}}$ éd., 1890, 208 p., p. 6.

29. FORSANT O. \& DUDOUIT P., op. cit.note 12, p. 31.

30. PIERRE A., LETRAIT L. \& M me BODIN Z, op. cit.note 1, p. 101.

31. GÉRARD J., op. cit.note 8, p. 122.

32. GÉRARD J., ibid.,p. 71.

33. JOST C. \& HUMBERT V., op. cit.note 3, p. 69.

34. DEVINAT E., op. cit.,note 1 . Cet échange, qui se situe à la veille de la première guerre mondiale, prend la forme d'un roman scolaire.

35. FORSANT O. \& DUDOUIT P., op. cit.note 12, préface.

36. PIERRE A., LETRAIT L. \& $\mathrm{M}^{\text {me }}$ BODIN Z., op. cit.note 1, p. 110.

37. Ibid.

38. PIERRE A., LETRAIT L. \& $\mathrm{M}^{\mathrm{me}}$ BODIN Z., op. cit.note 1, pp. 114-115.

39. DEVINAT E., op. cit.,note 1, pp. 23-25, 36-38, 43, 58 et 129. 
40. GÉRARD J., op. cit. note 8, p. 44.

41. PIERRE A., LETRAIT L. \& $\mathrm{M}^{\mathrm{me}}$ BODIN Z., op. cit.note 1, p. 93.

42. GÉRARD J., op. cit.note 8, pp. 133-137.

43. Octave AUBERT : La Patrie,cité in PIERRE A., LETRAIT L. \& $\mathrm{M}^{\mathrm{me}}$ BODIN Z., op. cit. note 1, p. 51. Voir aussi : THAUZIÈS [Max Ellyan] \& CAZALS, L'écolier ariègeois, récréations morales et littéraires Niveau non précisé,Pamiers, Librairie-imprimerie $\mathrm{M}^{\mathrm{me}}$ Delaye, 1900, 344 p., p. 329 ; ESTIENNE P. \& DANIEL H., op. cit.note 9, p. 37 etc.

44. GÉRARD J., op. cit.note 8, p. 179.

45. BAILLY J. \& DODEY C., La morale pratique de l'écolier - Cours élémentaire et Cours moyen,Paris, Éd.

H. Le Soudier, Nouvelle éd., 1919, 148 p., p. 55.

46. FORSANT O. \& DUDOUIT P., op. cit.note 12, p. 69.

47. BAUDRILLARD J. \& KUHN M., op. cit.note 16, pp. 32, 50 et 58.

48. DEVINAT E., op. cit.,note 1, p. 44.

49. ESTIENNE P. \& DANIEL H., op. cit.note 9, pp. 28-29.

50. DURAND H., «Conseils d'une abeille », cité in PIERRE A., LETRAIT L. \& $\mathrm{M}^{\mathrm{me}}$ BODIN Z., op. cit. note 1, p. 42 et p. 39.

51. CRESSOT J., L'éducation morale à l'école primaire - Niveau non précisé,Paris, Strasbourg, Librairie Istra, $3^{\mathrm{e}}$ éd., 1938, 192 p., p. 23.

52. BAUDRILLARD J. \& KUHN M., op. cit. note 16, p. 181.

53. BAUDRILLARD J. \& KUHN M., ibid.,p. 103.

54. ESTIENNE P. \& DANIEL H., op. cit.note 13, p. 11.

55. ESTIENNE P. \& DANIEL H., ibid.,p. 15.

56. BAUDRILLARD J. \& KUHN M., op. cit.note 19, p. 148.

57. GÉRARD J., op. cit.note 8, p. 22.

58. Conte nivernais adapté par MILLIEN A. et cité par BAUDRILLARD J. \& KUHN M., op. cit.note 16, p. 140.

59. BRUNO G., « Le livre de l'adolescent », cité in BAILLY J. \& DODEY C, op. cit.note 44, pp. 38-39.

60. JOST C. \& HUMBERT V., op. cit.note 3, pp. 111-112.

61. FORSANT O.\& DUDOUIT P., op. cit. note 12, p. 75.

62. FORSANT O. \& DUDOUIT P., ibid.,p. 137.

63. GÉRARD J., op. cit.note 11, p. 20-21.

64. Mais on trouve parfois des cas plus élaborés, mettant en scène des adultes, où la sanction est différée. Ainsi un conte moral, La revanche des arbres, dit le saccage d'une antique forêt vendue par des villageois cupides à un étranger, en dépit des conseils d'un sage instituteur ${ }^{(64)}$, et les conséquences désastreuses, à terme, de cet acte de barbarie.

65. GÉRARD J., op. cit.note 8, p. 141.

66. CUISSART E., Premier degré de lectures courantes - Niveau non précisé,Paris, Alcide Picard, vers



67. DEVINAT E., op. cit.,note 1, pp. 123-125 et GÉRARD J., op. cit.note 11, p. 132.

68. Cf. GUIBBERT P., «La mise en scène du Paradis dans les manuels de foi de la petite enfance », in Catéchismes et confessions de foi,Montpellier, Université Paul Valéry, 1995, 575 p., pp. 425-454.

69. Cf.NOËL F.-A., La nouvelle lecture rationnelle. Leçons morales, Leçons de choses - Niveau non précisé, Paris, Gedalge et $C^{\mathrm{ie}}, 71^{\mathrm{e}}$ éd., 1920, 141 p., p. 93 ; PIERRE A., LETRAIT L. \& $\mathrm{M}^{\text {me }}$ BODIN Z., op. cit. note 1,p. 114 ; GÉRARD J., op. cit.note 8, p. 6. ; BAUDRILLARD J. \& KUHN M., op. cit.note 16, p. 5 etc.

70. GÉRARD J., op. cit.note 8, p. 139. Lorsqu'il s'efforce de prendre le relais de l'école, le cinéma populaire des premiers temps privilégie cet aspect répressif. Cf.DE LA BRETÈQUE F. \& GUIBBERT P., «Mission moralisatrice et romantisme social du cinéma des premiers temps », in Les vingt premières années du cinéma français,Paris, Presses de la Sorbonne Nouvelle, A.F.R.H.C., 1996, pp. 223-248. 
71. Cf.LEBAIGUE Ch., Le livre de l'école - Cours préparatoire,Paris, E. Belin, 1885, 114 p., p. 33 STEEG J., Instruction morale et civique - Niveau non précisé,Paris, N. Fauvé, F. Nathan, 1882, 179 p., p. 27.

72. MÉZIÈRES M. A., Éducation morale et instruction civique - Cours moyen et Cours supérieur,Paris, Ch. Delagrave, 1883, 5 éd., 1893, 176 p., p. 92 ; GÉRARD J., op. cit.note 8, p. 139.

73. Cf.BILLARD C. \& GUIBBERT P., Le petit catéchisme illustré ou la fin d'un mythe, Paris, Belfond, 1976, 276 p. BILLARD C. \& GUIBBERT P., On nous a changé la religion! Apprendre Dieu hier et aujourd'hui. Paris, Arléa, Corlet, 1997, 191 p.

74. BAUDRILLARD J. \& KUHN M., op. cit.note 16.

75. NOËL F.-A., op. cit. note 68.

\section{RÉSUMÉS}

Non disponible

Not available

INDEX

Mots-clés : école primaire, IIIe république, morale, ruralité

Keywords : morality, primary school, rural context, rural life, third republic

\section{AUTEUR}

\section{PIERRE GUIBBERT}

Directeur du CEDRHE, IUFM de Montpellier 